\title{
PRONTUÁRIO ELETRÔNICO DE ESTOMATOLOGIA (PEEST): UMA EXPERIÊNCIA DE DESENVOLVIMENTO E IMPLANTAÇÃO NO CURSO DE ODONTOLOGIA
}

\section{ELECTRONIC PROMOTION OF STOMATOLOGY (PEEST): AN EXPERIENCE OF DEVELOPMENT AND IMPLANTATION IN THE DENTISTRY COURSE}

Recebido em: 29/09/2018.

Aceito em: 16/11/2018.

\author{
Danilo Rangel Arruda Leite ${ }^{1}$ \\ Alisson de Oliveira Silva ${ }^{2}$ \\ Gustavo Brito Sampaio ${ }^{3}$ \\ Lino João da Costa ${ }^{4}$ \\ Paulo Rogério Ferreti Bonan ${ }^{5}$ \\ Cláudia Batista Mélo'
}

\section{RESUMO}

Este trabalho visa apresentar uma experiência de participação colaborativa no processo de desenvolvimento, validação, análise de qualidade e usabilidade do Prontuário Eletrônico de Estomatologia (PEEst), no curso de Odontologia da Universidade Federal da Paraíba (UFPB). O PEEst foi implementado a partir de uma metodologia de desenvolvimento e validação estruturada em etapas, utilizando a linguagem de programação PHP, JavaScript, HTML5 e CSS. Os testes e validação contaram com a participação de docentes e discentes da disciplina de Estomatologia II, que

\footnotetext{
1 Doutorando em Modelos de Decisão e Saúde pela Universidade Federal da Paraíba (UFPB). Analista do setor de Pesquisa e Inovação Tecnológica do Hospital Universitário Lauro Wanderley (HULW), Empresa Brasileira de Serviços Hospitalares (EBSERH). E-mail: danilorangel@buscapb.com.br
}

2 Mestre em Estatística pela Universidade Federal de Pernambuco (UFPE). Analista do setor de Pesquisa e Inovação Tecnológica do Hospital Universitário Lauro Wanderley (HULW), Empresa Brasileira de Serviços Hospitalares (EBSERH).

E-mail: alisson.oliveira@ebserh.gov.br

3 Graduando em Ciência da Computação pela Universidade Federal da Paraíba (UFPB). E-mail: gbritosampaio@gmail.com

4 Doutor em Odontologia pela Universidade de São Paulo (USP). Professor Titular da Universidade Federal da Paraíba (UFPB). E-mail: linojcosta@gmail.com

5 Doutor em Estomatopatologia pela Universidade Estadual de Campinas (UNICAMP). Professor Adjunto da Universidade Federal da Paraíba (UFPB). E-mail: pbonan@yahoo. com

6 Doutora em Engenharia Elétrica pela Universidade Federal de Campina Grande (UFCG). Professora Associada da Universidade Federal da Paraíba (UFPB).

E-mail: claudiabmelo@gmail.com 
registraram as informações no PEEst e responderam ao formulário de avaliação. Foi verificada a necessidade de capacitações dos usuários com relação a qualidade dos registros das informações. O PEEst mostrou ser uma ferramenta eficaz com interface autoexplicativa, permitindo acesso seguro, possibilitando registrar fotos e informações detalhadas do paciente e de suas lesões, sendo uma ótima opção para ser utilizada nas Clínicas de Estomatologia, especialmente, nas clínicas escolas.

Palavras-chave: Medicina bucal. Sistemas computadorizados de registros médicos. Validação de software.

\section{ABSTRACT}

This work aims to present a collaborative participation in the development process, validation, analysis of quality and usability of electronic health record of Stomatology (PEEst) in the course of dentistry from the Federal Univer sity of Paraíba(UFPB). The PEEst was implemented from a development and validation methodology structured in steps, using the programming language PHP, JavaScript, Html and CSS. The testing and validation with the participation of teachers and students of Stomatology II, who recorded the information on PEEst and responded to the evaluation form. It was verified the need for training of users regarding the quality of the records of the information. The PEEst has proved to be an effective tool with self-explanatory interface, allowing for secure access, enabling register photos and detailed information of the patient and of his injuries, being a great option for use in Dental clinics, especially in clinics schools.

Keywords: Oral Medicine. Medical Records Systems, Computerized. Software Validation.

\section{INTRODUÇÃO}

Os cursos de Bacharelado em Odontologia visam a formação de profissionais para atuar na saúde e estética bucal, com vivência em atendimento odontológico, de forma competente, para tomar decisões e com capacidade de buscar educação continuada quanto aos conhecimentos inerentes à prática odontológica. Para a construção do diagnóstico e, consequentemente, do desenvolvimento do plano de tratamento, o Cirurgião Dentista faz uso do prontuário odontológico como ferramenta de coleta, observação e interpretação dos dados do paciente (DITTERICH et al., 2008).

Durante o curso de bacharelado em Odontologia, o prontuário odontológico é fundamental nas disciplinas de clínica, sendo a Clínica de Estomatologia o primeiro contato entre o estudante e o paciente (BENEDICTO et al., 2010). 
O prontuário odontológico é um documento que registra informações do atendimento odontológico do paciente. Dentre as informações registradas nesse prontuário, podemos destacar: anamnese, história médica, doenças de infância, uso de medicações, hábitos pessoais, história odontológica e odontograma (MAGNAGNAGNO, 2015).

O prontuário odontológico em papel apresenta várias desvantagens como, por exemplo, limitações na guarda e no acesso. Com o prontuário eletrônico odontológico é possível prover segurança aos dados, armazenar muito mais informações ocupando pouco espaço, agilizar e otimizar os procedimentos de inclusão das informações do paciente, disponibilizar a informação a qualquer tempo e em qualquer lugar; controlar a autenticidade, impedir adulterações, garantir o sigilo dos dados do paciente entre outros (PEREIRA; PAIVA, 2011).

Diante do exposto, este trabalho tem como objetivo apresentar o PEEst, Prontuário Eletrônico de Estomatologia, desenvolvido para Clínica de Estomatologia da Universidade Federal da Paraíba (UFPB). No caso de clínicas escolas, o PEEst visa atender às necessidades inerentes às disciplinas de Estomatologia por apresentar uma alternativa que dará aos docentes e discentes uma ferramenta auxiliar para tornar o atendimento mais padronizado, orientado, sistematizado e ágil, possibilitando o acesso restrito e controlado de todo o tratamento.

Para o desenvolvimento do PEEst, algumas características foram levadas em consideração, tais como: ser multiplataforma, reduzir custos com recursos de informática, agilidade de implantação, interface simples e amigável (PEREIRA; PAIVA, 2011).

\section{MÉTODO}

A informatização da Clínica de Estomatologia, da disciplina Estomatologia do curso de Odontologia da UFPB teve seu início em 2016. A implementação do PEEst tem a participação de uma equipe multidisciplinar que conta com docentes, discentes e profissionais da Odontologia e Computação.

Para o desenvolvimento do PEEst foi utilizada a linguagem de programação PHP, JavaScript, HTML5 e CSS com Banco de Dados MySQL. A escolha dessas ferramentas se deu pelo fato de serem linguagens de desenvolvimento Web, possibilitando o acesso a partir de dispositivos móveis, além de permitir a programação de aplicações responsivas (que se adaptam ao tamanho da tela) (YEN; BAKKEN, 2012), promovendo interoperabilidade entre os principais navegadores e sistemas operacionais de dispositivos móveis disponíveis na atualidade, tais como iOs e Android (COMSCORE, 2011; SILVEIRA, 2010; SOTO, et al., 2006).

A metodologia de desenvolvimento e validação do PEEst foi estruturada nas seguintes etapas: mapeamento dos fluxos de trabalhos, identificação e homologação de análise e requisitos do PEEst, instrumento de validação e avaliação da utilização do PEEst (BRAGA, 2013). 


\section{MAPEAMENTO DOS FLUXOS DE TRABALHOS}

O mapeamento foi feito com base na definição de fluxos de trabalho onde a circulação de documentos e/ou tarefas é realizado por meio de um processo de trabalho, ou seja, o aspecto operacional de uma metodologia de trabalho: quem as executa, suas prioridades de execução, como as tarefas são organizadas, como são sincronizadas, como estas tarefas estão sendo acompanhadas (SILVEIRA, 2010).

Foi idealizado um fluxograma para a divisão do desenvolvimento das partes do sistema. Este foi apresentado a todos da equipe (desenvolvedores, professores e colaboradores), tendo grande importância pois, a partir dele, foi definida a ordem e cronograma de desenvolvimento do PEEst.

Além do desenvolvimento do sistema, foram pesquisadas informações adicionais como a existência de outros prontuários eletrônicos odontológicos para identificar características que pudessem ajudar nas opções do PEEst.

Os principais responsáveis por cada processo que envolvia os procedimentos do atendimento dos pacientes da clínica se reuniram em vários momentos para a criação dos fluxos ao longo de um semestre. Colaboradores da área da Tecnologia da Informação (TI) foram responsáveis pela tradução dos fluxos para notação BPMN (OMG, 2017).

\section{IDENTIFICAÇÃO E HOMOLOGAÇÃO DE ANÁLISE E REQUISITOS DO PEEST}

Reuniões foram realizadas entre docentes, discentes, analistas, desenvolvedores e colaboradores da UFPB com o objetivo de identificar e homologar requisitos para o PEEst. A equipe de desenvolvimento do sistema discutiu no início do projeto a forma de nomear as variáveis utilizadas. Sendo esse um grande problema em qualquer trabalho envolvendo linguagens de programação (BRAGA et al., 2013), por isso, resolvemos adotar um padrão predefinido de nomeclatura das variáveis para não haver problemas à frente.

Essa padronização facilitou a criação do banco de dados do sistema e as modificações feitas no código-fonte, sendo possível encontrar os campos a serem modificados mais rapidamente, para que os futuros relatórios das informações coletadas pudessem ajudar no processo de tomada de decisão de planejamento e pesquisa. Durante um ano, as reuniões para levantamentos de requisitos, foram realizadas uma vez por semana, com duração aproximada de 3 horas.

Por fim, para manter o código fonte constantemente atualizado, foi utilizado o sistema online GitHub (serviço web gratuito que oferece hospedagens para projetos de software e permite o controle de versões do sistema), para organização e divisão de tarefas dentro do grupo ou empresa, foi utilizado o Runrun.it (software gratuito, utilizado como gerenciador de tarefas, tempo e desempenho da equipe) e o GoogleDrive (serviço online que permite o armazenamento de arquivos na nuvem do Google), utilizado para compartilhar os relatórios com todos os participantes do projeto. 


\section{INSTRUMENTO DE VALIDAÇÃO DO PEEST}

O PEEst é um sistema on-line que permite registrar, armazenar e acessar informações do paciente, auxiliando o processo de tomada de decisão na conduta a ser seguida pelo estomatologista e/ou estudante para cada paciente (Figura 1).

Figura 1 - Tela de cadastro de identificação do paciente, 2017.

\section{(1) PEESt}

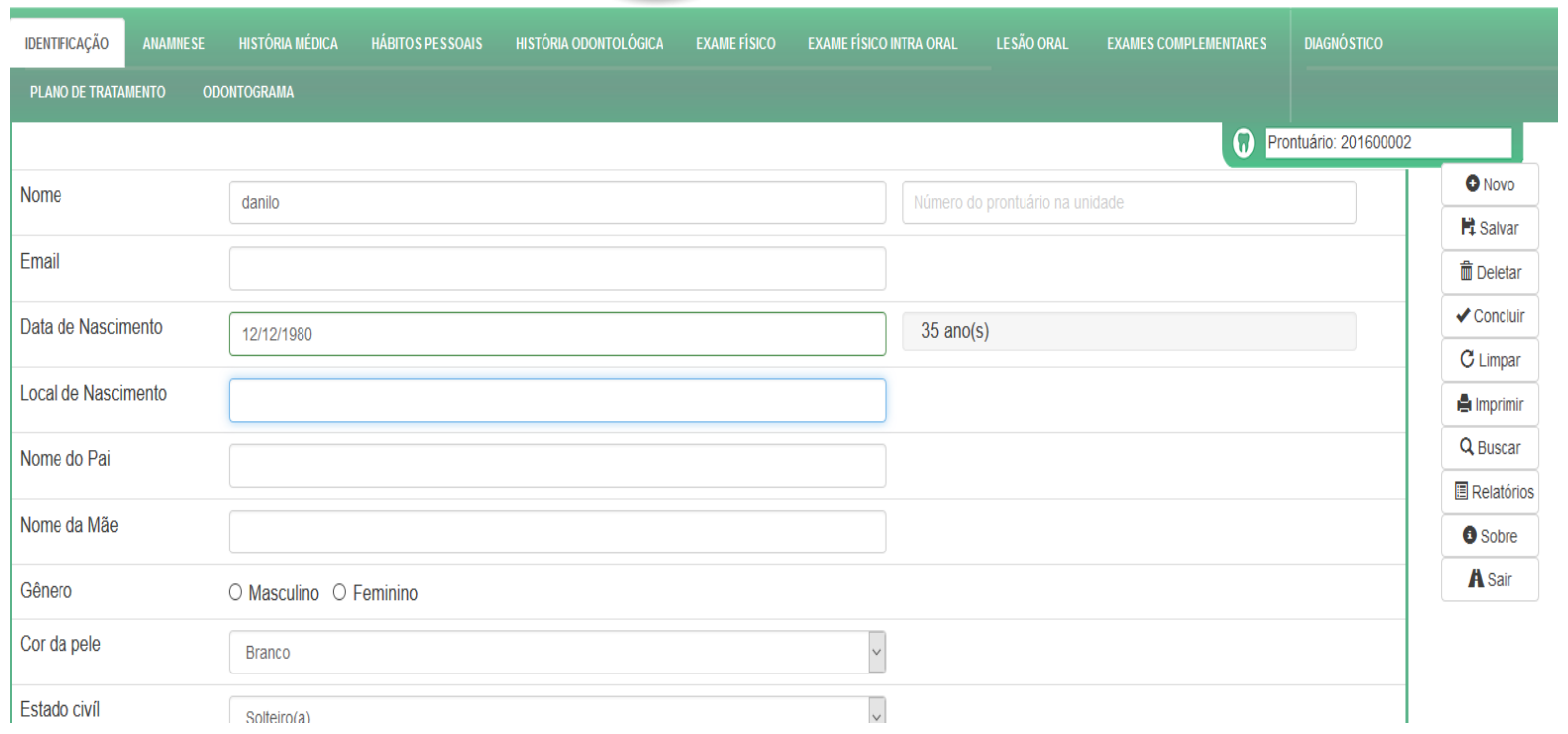

Os testes do PEEst em dispositivos móveis foram feitos através de duas ferramentas, o Phonegap Framework que dá melhor suporte a criação para esses dispositivos com as linguagens escolhidas, e o Apache Ripple que simula um ambiente de aparelho móvel, para evitar que cada mudança precise ser testada diretamente em cada dispositivo. $\mathrm{O}$ acesso ao sistema foi disponibilizado por meio da plataforma web, através do link temporário peest.buscapb.com.br e, para tal, os usuários utilizaram login e senha cadastrados previamente pelo admistrador do sistema.

Embora existam softwares odontológicos comerciais, principalmente, para auxiliar a gestão de clínicas e consultórios, são poucos os exemplos de sistemas com foco na área de Estomatologia e que estejam voltados para o meio acadêmico (PINTO, 2006).

Algumas das principais características do PEEst são:

- $\quad$ está disponível na plataforma Web, podendo ser usado a qualquer hora e de qualquer lugar com acesso à Internet; 
- dispõe de diferentes visões conforme o tipo de usuário (dentistas, docentes, discentes, atendentes e administrador do sistema);

- multiplataforma e responsivo, adaptando-se a computadores e dispositivos móveis;

- $\quad$ pode ser usado em ambientes acadêmicos, com a liberação do paciente condicionada à análise e aprovação do docente supervisionador;

- desenvolvido especificamente para Estomatologia, proporcionando uma detalhada descrição de lesões bucais, armazenamento de exames, incluindo os fotográficos;

- $\quad$ apresenta vários modelos de relatórios pré-definidos e disponibiliza a opção de geração dinâmica, permitindo ao usuário elaborar e customizar seus próprios relatórios;

- não é necessário fazer a sua instalação no computador ou dispositivo móvel;

- $\quad$ agilidade na coleta, organização e acesso às informações.

Vale ressaltar que os prontuários eletrônicos odontológicos devem possuir um sistema de segurança forte com senhas criptografadas que limitem o acesso a usuários autorizados e forneçam uma política de backup que assegure o armazenamento dos dados, protegendo pacientes e profissionais (PRONTUÁRIO, 2012). Entretanto, o sistema de backup ainda está em fase de desenvolvimento e entrará em produção nos próximos meses.

Ao longo do desenvolvimento do PEEst, docentes e colaboradores realizaram os testes de validação utilizando dispositivos móveis e computadores pessoais em busca de melhorias. Para isso, foi criado um questionário estruturado, como instrumento de coleta de dados para validar o PEEst, contendo dez questões relacionadas às funcionalidades e à usabilidade do sistema.

Dentre os dados registrados no PEEst, podemos destacar: dados pessoais, anamnese, história médica, doenças de infância, uso de medicações, hábitos pessoais, história odontológica, exame físico, odontograma, exames complementares, diagnóstico, lesão orla e plano de tratamento. A seguir, serão apresentadas algumas das telas do PEEst e suas funções.

A Figura 2 mostra a opção de exames complementares, onde o profissional poderá armazenar exames e fotos que o ajudaram no diagnóstico. 
Figura 2 - Tela de inclusão de exames fotográficos, 2017.

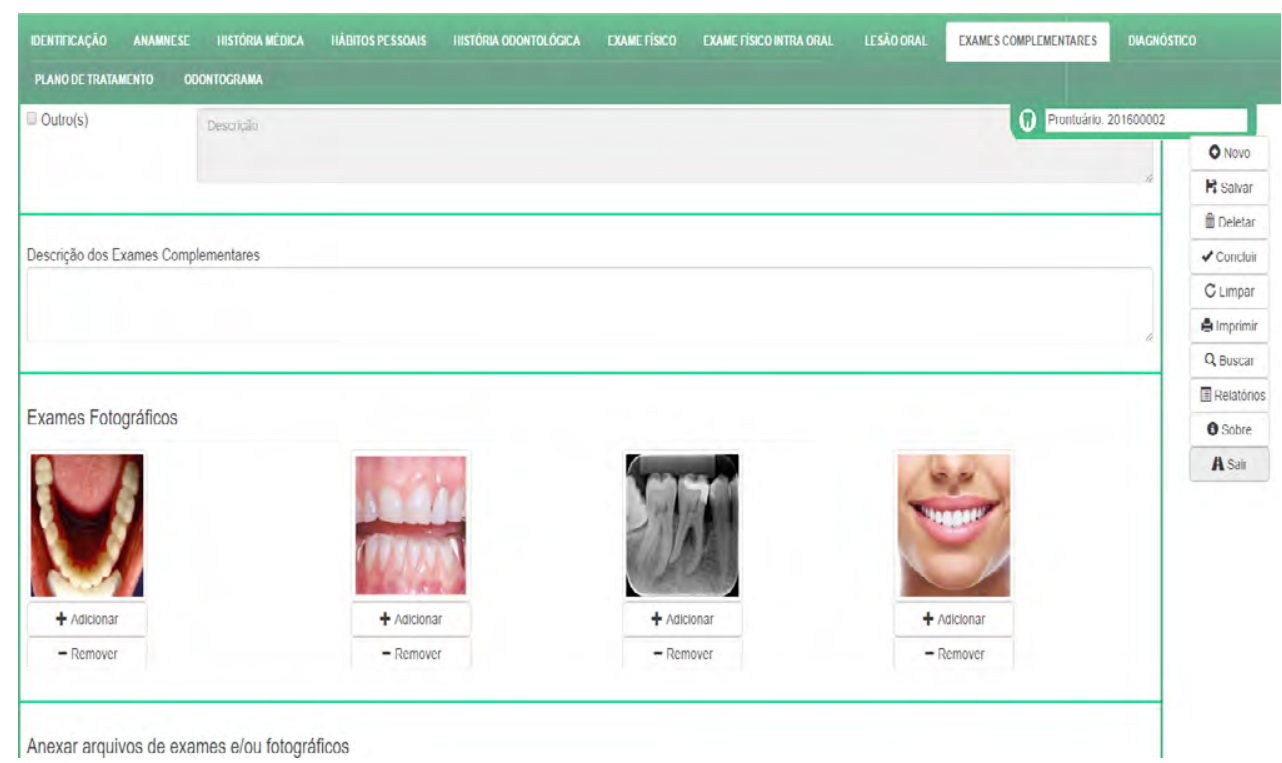

A Figura 3 mostra a tela de Lesão Oral, sendo, esta opção, o maior difencial entre o PEEst e os outros Prontuários Eletrônicos Odontológicos existentes, pois no PEEst o profissional descreverá detalhadamente e estruturadamente as lesões bucais encontradas no paciente.

Figura 3 - Tela de Lesão Oral, 2017.

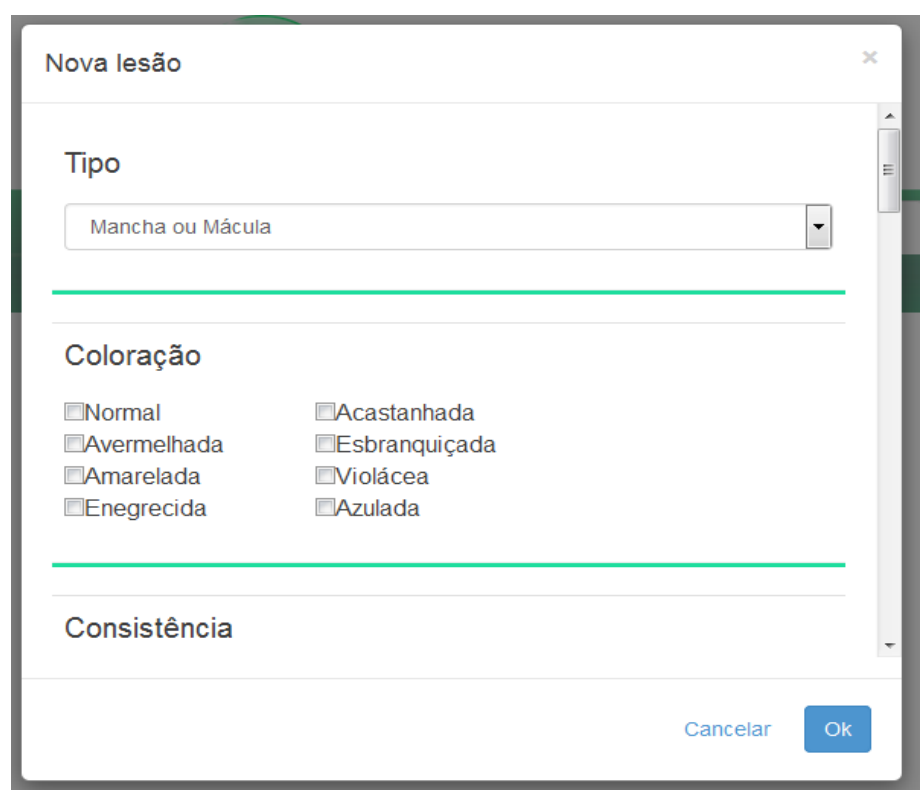

Na Figura 4, Odontograma, é possível incluir diagnósticos endodônticos e dentários de cada uma das faces dos dentes, bem como, iniciar cada um dos procedimentos descritos no Plano de Tratamento. Sendo, esse, a representação gráfica da boca do paciente. Estão dispostos no odontograma os dentes decíduos e definitivos. 
Figura 4-Odontograma, 2017.

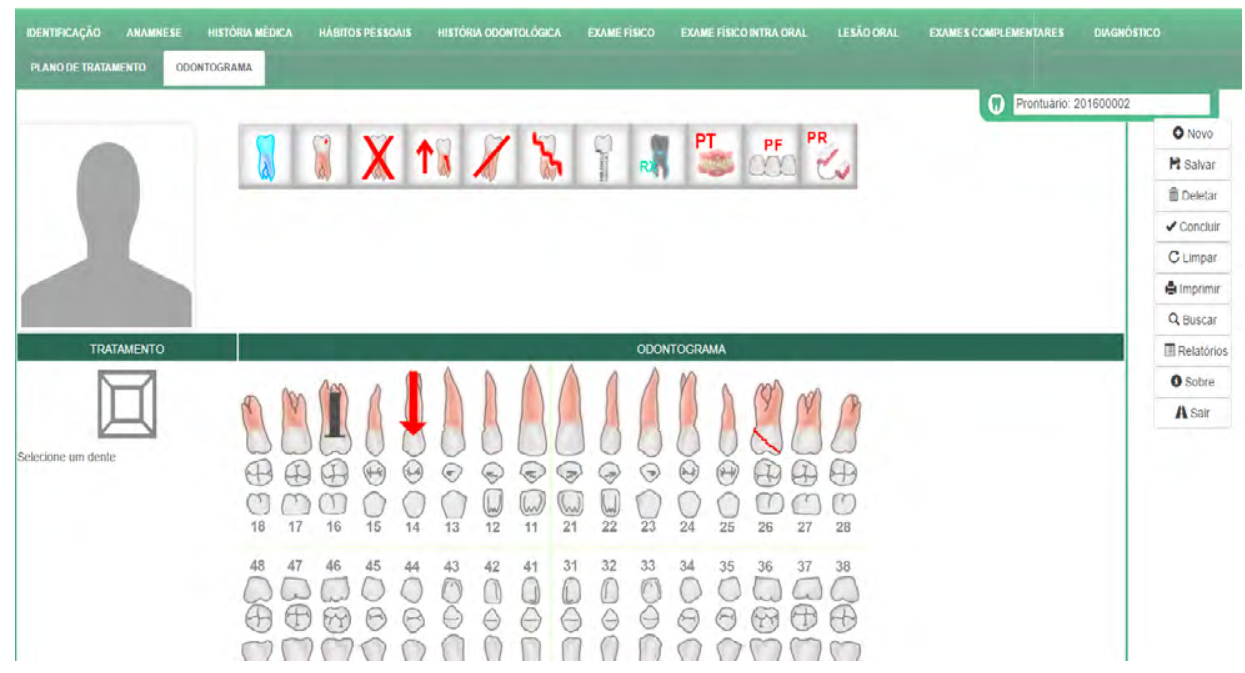

\section{Avaliação da utilização do PEEst}

Nesta fase, o sistema foi submetido a testes de uso por docentes e discentes em diferentes equipamentos, tais como, smartphones, Tablets e computadores pessoais.

O estudo exploratório foi realizado na Clínica de Estomatologia da UFPB. Os primeiros testes de validação do PEEst visaram avaliar a usabilidade sob os apectos de facilidade de uso, tempo de carregamento, adequação a resolução da tela, frequência de uso e relevância dos dados e foram planejados e divididos em duas fases: Teste e Validação Docente e Teste e Validação Discente.

\section{Teste e Validação Docente}

Para os testes de validação realizados pelos docentes do curso de Bacharelado em Odontologia da UFPB, na Clínica de Estomatologia desta instituição, de forma voluntária, os docentes participaram desta etapa, com agendamento de acordo com a sua disponibilidade.

Cada voluntário docente recebeu um $i \mathrm{Pad}$ e instruções prévias sobre como seria a metodologia dos testes, bem como um prontuário em papel do paciente em tratamento na Clínica de Estomatologia, cujos dados da anamnese dos pacientes deveriam ser transcritos para o PEEst (CANÊ; RONDINA, 2014).

O docente era então acompanhado por dois discentes durante todo procedimento, os quais tinham a função de auxiliar o docente, registrando suas respostas relacionadas às perguntas do questionário de validação. A atuação dos docentes era concentrada apenas no preenchimento do PEEst e a ajuda dos monitores (discentes) no registro sistemático das respostas no instrumento de validação. 


\section{Teste e Validação Discente}

Os testes de validação realizados pelos discentes do curso de Bacharelado em Odontologia da UFPB também foram realizados na Clínica de Estomatologia, conforme ilustra a Figura 5, ambiente em que os alunos já possuíam familiaridade por atuarem durante suas práticas clínicas.

Figura 5 - Cenário de testes de utilização e apresentação do PEEst, 2017.

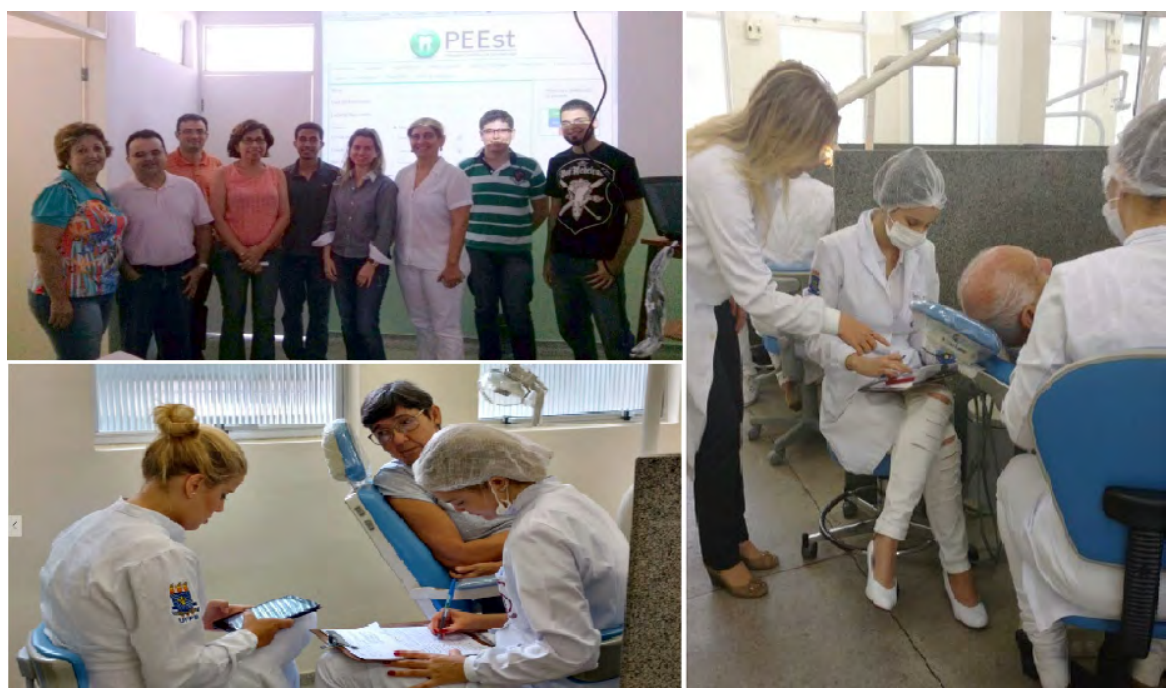

As turmas de discentes que estavam cursando ou já haviam cursado a disciplina Estomatologia II participaram como voluntários dos teste e validação do PEEst. Os discentes ficaram responsáveis pelo preenchimento dos dados do paciente no PEEst e do questionário de validação. Os mesmos foram divididos em dois grupos: (a) o primeiro grupo utilizou os prontuários em papel de pacientes já preenchidos e apenas transferiu as informações para o PEEst; (b) o segundo grupo registrou diretamente no PEEst os dados fornecidos pelo paciente, durante a anamnese. Os dois grupos utilizaram o mesmo instrumento de validação utilizado pelos docentes. Os testes foram realizados durante um semestre.

Ao final da fase de validação e testes, os discentes afirmaram que a experiência com o PEEst foi estimulante, dinâmica, prática e proveitosa, além de ser bem mais completo e proporcionar uma experiência mais didática que o prontuário em papel. A interface foi considerada atrativa, amigável e de fácil utilização. Foi relatado que o sistema tem uma navegação bem elaborada, não sendo considerado cansativo.

Alguns aluno(a)s do $5^{\circ}$ período de Odontologia que participartam dos testes, deram os seguintes depoimentos:

"Eu achei o PEEst muito completo, agiliza muito o atendimento ao paciente, posso incluir os dados com o meu Tablet ou notebook."

"Achei a interfece muito fácil de navegar!" 


\section{RESULTADOS E DISCUSSÕES}

Os testes e validação do PEEst contaram com a participação de 06 docentes e 47 discentes, da disciplina Estomatologia II que registraram as informações no PEEst e responderam ao formulário de avaliação. Considerando o número total de docentes do quadro efetivo (n=53) do Departamento de Clínica e Odontologia Social da UFPB e de discentes dos 10 períodos da graduação $(n=400)$, a participação representou em torno de $11 \%$ dos docentes e $11 \%$ dos discentes.

Durante os testes, foi constatada a necessidade de capacitações periódicas dos usuários com relação ao registro das informações, pois há uma tendência de falta de qualidade do registro da informação e erros frequêntes, seja no prontuário em papel ou digital (BRAGA, 2013).

Em relação a utilização do PEEst, os docentes passam a: (a) se comprometer a se tornar um multiplicador dentro do processo de capacitação, e (b) exigir dos discentes o registro de informações com mais qualidade no sistema.

Das dez questões de cada um dos 53 formulários respondidos, pelos docentes e discentes, as duas que de forma mais clara revelam a utilidade e facilidadede de uso do sistema (DITTERICH et al., 2008) foram selecionadas para a discussão dos seus resultados: O PEEst atende as necesidades da Clínica de Estomatologia?; O PEEst é uma ferramenta fácil de usar?. A Figura 6 ilustra graficamente as respostas dessas questões respondidas. Para cada declaração, foi utilizada uma escala Likert (LIKERT, 1932) de seis pontos, tendo para cada uma das alternativas um valor numérico definido, sendo estes: discordo fortemente $=1$; discordo $=2$; discordo levemente $=3$; concordo levemente $=4 ;$ concordo $=5$; concordo fortemente $=6$.

Figura 6 - Respostas obtidas nas questões escolhidas para a discussão da qualidade de uso do PEEst, 2017.
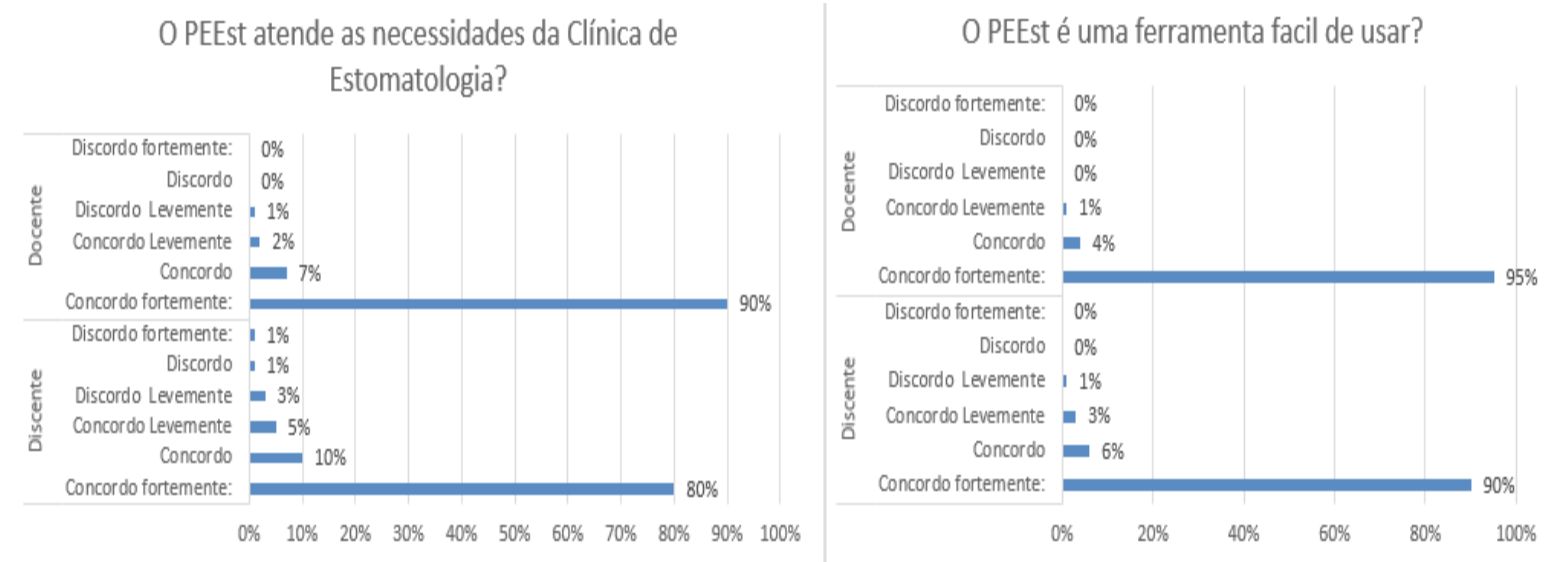

Contudo, o sistema foi considerado completo e adequado para a Estomatologia, agradável e flexível para ser utilizado em qualquer dispositivo, seja móvel ou não. 
Dentre as desvantagens relatadas, os participantes citaram a dependência da internet, não poder utilizar o sistema em dispositivos móveis off-line e a ausência de um sistema de bacukp.

\section{CONCLUSÃO}

Este trabalho apresentou o Prontuário Eletrônico de Estomatologia (PEEst) utilizado no curso de Bacahrelado em Odontologia na Universidade Federal da Paraíba (UFPB). Como ainda está em fase de ajustes e melhorias, a validação do PEEst, no seu estágio final de desenvolvimento, possivelmente apresentará resultados distintos dos apresentados nesse documento. Porém, considerada a complexidade de um sistema voltado para à assistência e para o ensino (DITTERICH et al., 2008), o planejamento da validação em um ambiente propício e apropriado, visando o engajamento dos usuários finais, não somente na validação como também durante todo processo de desenvolvimento do sistema, pôde mostrar a importância dessa participação colaborativa em busca de uma melhor qualidade para o sistema desenvolvido, assim como a necessidade de uma constante capacitação dos envolvidos para uma melhor qualidade do registro da informação (GODOY et al., 2012).

Por fim, o PEEst mostrou ser uma ferramenta eficaz com interface autoexplicativa, permitindo acesso a qualquer hora e de qualquer lugar, seguro e fácil, possibilitando, ainda, registrar fotos e outras infrmações detalhadas do paciente e de suas lesões, sendo uma ótima opção para ser utilizada nas Clínicas de Estomatologia.

\section{REFERÊNCIAS}

DITTERICH, R. G. et al. A importância do prontuário odontológico na clínica de graduação em Odontologia e a responsabilidade ética pela sua guarda. Rev Inst Ciênc Saúde, v. 26, n. 1, p. 120-4, 2008.

BENEDICTO, E. D. N. et al. A importância da correta elaboração do prontuário odontológico. Revista Literatura, v. 18, n. 36, p. 41-50, 2010.

MAGNAGNAGNO OA. Mecanismos de proteção da privacidade das informações de prontuário eletrônico de pacientes de instituições de saúde. 2015.

PEREIRA, S. R.; PAIVA, P. B. A importância da Engenharia da Usabilidade para a Segurança de Sistemas Informatizados em Saúde. J. Health Inform, v. 3, n. 3, p. 123-9, Julho-Setembro 2011. 
COMSCORE REPORTS February 2011 U.S. Mobile Subscriber Market Share, 2011. Disponivel em: <http://www.comscore.com/Insights/Press-Releases/2011/4/ comScore-Reports-February-2011-US-Mobile-Subscriber-Market-Share $>$. Acesso em: 25 março 2016.

SILVEIRA, D. T. et al. Sistema Nursing Activities Score: etapas de desenvolvimento de um sistema móvel para enfermagem. J. Health Inform, v. 2, n. 2, p. 44-50, AbrilJun 2010.

OMG. Documents Associated With BPMN, 2014. Disponivel em: <http://www. omg.org/spec/BPMN/2.0.2/>. Acesso em: 24 março 2016.

BRAGA, R. D. et al. Validação do prontuário eletrônico do paciente em uma instituição de ensino superior em saúde: relato da experiência no módulo Anamnese. J. Health Inform, v. 5, n. 1, p. 30-5, Janeiro-Março 2013.

PINTO, V. B. Prontuário eletrônico do paciente: documento técnico de informação e comunicação do domínio da saúde. Enc. Bibli: R. Eletr. Bibliotecon. Ci. Inf., Florianópolis, v. 11, n. 21, 2006.

LIKERT, R. A Technique for the Measurement of Attitudes. Archives of Psychology. 140. ed. New York: The Science Press, v. 22, 1932. 1-55 p.

CANÊ, P. K.; RONDINA, J. M. Prontuário Eletrônico do Paciente: conhecendo as experiências de sua implantação. J. Health Inform, v. 6, n. 2, p. 67-71, Abril-Junho 2014.

GODOY, J. S. D. M. et al. O uso do prontuário eletrônico por enfermeiros em Unidades Básicas de Saúde brasileiras. J. Health Inform, v. 4, n. 1, p. 3-9, Janeiro-Março 2012.

13 YEN, P. Y.; BAKKEN,. Review of health information technology usability study methodologies. J Am Med Inform Assoc, 19, n. 3, May-Jun $2012.413-422$.

SOTO, et al. Communication in critical care environments: mobile telephones improve patient care. Anesth Analg, 102, n. 2, Feb 2006. 535-41.

PRONTUÁRIO Eletônico - A certificação de Sistemas de Registro Eletrônico de Saúde. Sociedade Brasileira de Informática em Saúde - SBIS, 2012. Disponivel em: <http://www.sbis.org.br/certificacao/Cartilha_SBIS_CFM_Prontuario_ Eletronico_fev_2012.pdf>. Acesso em: 20 mar 2016. 